\title{
APORTACIÓN AL CONOCIMIENTO CARIOLÓGICO DEL GÉNERO RANUNCULUS L. SUBGÉNERO FICARIA (SCHAEFFER) L. BENSON EN LA PENÍNSULA IBÉRICA
}

\author{
Juan Carlos DIOSDADO y Julio PASTOR
}

\begin{abstract}
RESUMEN. Aportación al conocimiento cariológico del género Ranunculus L. subgénero Ficaria (Schaeffer) L. Benson en la Península Ibérica. Se realiza un estudio cariológico de $R$. ficaria L. en la Península Ibérica. Se ha encontrado principalmente el número somático $2 \mathrm{n}=16$, aunque algunas poblaciones son triploides $(2 n=24)$ o tetraploides $(2 n=32)$. A partir de los datos cromosómicos (cariogramas, idiogramas, índices de asimetría y niveles de ploidía) se han establecido relaciones entre los distintos citotipos.
\end{abstract}

Palabras clave. Cariología, Ranunculus, Ficaria, Península Ibérica.

SUMMARY. Contribution to the kariological study of the genus Ranunculus L. subgenus Ficaria (Schaeffer) L. Benson from the Iberian Peninsula. A caryological study of $R$. ficaria L. from the Iberian Peninsula has been made. The somatic number is $2 n=16$, although some populations have triploids $(2 n=24)$ or tetraploids $(2 n=32)$. From the caryological data (caryograms, idiograms, asymmetry indices and polyploid levels) relationships have been established between the cytotypes.

Key words. Karyology, Ranunculus, Ficaria, Iberian Peninsula.

\section{INTRODUCCIÓN}

EL subgénero Ficaria está integrado en la Península Ibérica por $R$. ficaria $\mathrm{L}$. que se distribuye por Europa, zonas del oeste y centro de Asia y norte de Africa. Debido a ciertas características distintivas, como la presencia de 3 sépalos y 7-14 pétalos, ha sido considerado por algunos autores como género independiente. Haller (1742) consideró Ficaria como género, según el concepto de Dillenius. Sin embargo, Linneo (1753) lo incluyó en el género Ranunculus como $R$. ficaria L. Posteriormente, Schaeffer (1760) adoptó el criterio de Haller. Muchos autores posteriores aceptaron la separación de Ficaria y Ranunculus, como por ejemplo Jussieu (1789), De Candolle (1824), Spach (1839), Freyn (1880), Ovczinnikov (1937) o Tamura (1967). Sin embargo, actualmente se acepta que Ficaria forma parte del género Ranunculus. Boissier (1867) lo consideró como sección dentro de 
Ranunculus, siendo esto admitido por algunos autores como Tutin (1964). No obstante, Benson (1940) consideró a Ficaria como subgénero, y este criterio ha sido aceptado por la mayoría de los autores actuales: Maire (1964), López González (1986) o Valdés (1987). Según Tutin (1964), R. ficaria está representado en la Península Ibérica por las subespecies ficaria, ficariiformis Rouy \& Fouc. y bulbifer Lawalrée. López González (1986) considera la subsp. ficariiformis dentro de la subsp. ficaria, indicando, no obstante, que aquellos ejemplares más robustos, con flores mayores, etc, correspondería a lo que otros autores atribuyen a la primera subespecie.

Dado que en la extensa bibliografía cariológica consultada (tab. 1) no se indica, en la mayoría de los casos, a que subespecie corresponden los datos aportados, se exponen más adelante de modo conjunto las observaciones de los 3 niveles (diploide, triploide y tetraploide) encontrados, en el presente estudio, para la Península Ibérica. Las plantas diploides $(2 n=16)$ corresponden a la subsp. ficaria, pues al estudiar los cariogramas en plantas de morfología variable no se observó ninguna diferencia significativa, lo que apoyaría, bajo una perspectiva cariológica, el tratamiento taxónómico de López González (1.c.). Las plantas tetraploides $(2 \mathrm{n}=32)$ pertenecen a la subsp. bulbilifer Lambinom (=subsp. bulbifer Lawalrée), caracterizada por la presencia de hojas provistas de bulbillos axilares. Por último, las plantas triploides $(2 n=24)$ muestran una posición taxonómica incierta, aunque la ausencia de aquenios fértiles y de bulbillos axilares apuntan hacia un origen híbrido entre diploides y tetraploides.

Por otro lado, cabe destacar que a pesar de los numerosos datos carịológicos de $R$. ficaria existentes en la bibliografía, muy pocos corresponden a poblaciones de la Península Ibérica: Barros Neves (1942), Löve \& Kjellqvist (1974), Valdés-Bermejo (1979), Pogan \& Wcislo (1986) y Luque et al. (1988).

\section{MATERIAL Y MÉTODOS}

Las observaciones de las meiosis se realizaron a partir de botones florales fijados en el campo con líquido de Farmer, alcohol etílicocloroformo-ácido acético en proporción 6:3:1 (Löve \& Löve, 1975), durante 24 horas, tras las cuales se pasaron a alcohol etílico al $70 \%$ donde se conservaron hasta su tinción. Esta se realizó con carmín clorhídrico etílico (Snow, 1963) durante 72 horas. Posteriormente las anteras se montaron por aplastamiento en ácido acético al $45 \%$.

Los estudios de cromosomas en mitosis se llevaron a cabo en meristemos radicales de plantas cultivadas en el jardín experimental del Departamento de Biología Vegetal y Ecología de la Facultad de Biología de Sevilla. Este material fue pretratado con 8hidroxiquinoleína $0.002 \mathrm{M}$. (Tjio \& Levan, 1950) a $4 \pm 2{ }^{\circ} \mathrm{C}$ durante $3-4$ horas. A continuación se fijaron en Carnoy (Löve \& Löve, 1975) durante 24 horas, y despues se conservaron en alcohol al $70 \%$. La tinción se realizó con carmín clorhídrico etílico durante 48-72 horas. El montaje se efectuó en ácido acético al $45 \%$.

Para la morfología de los cromosomas se ha considerado la clasificación de Levan et al. (1964). Para la clasificación de los cromosomas por su tamaño se ha seguido a Stebbins (1938). La asimetría de los cariotipos se define de acuerdo con las indicaciones de Stebbins (1971), utilizándose además los índices de asimetría $\left(\mathrm{A}_{1}\right.$ y $\left.\mathrm{A}_{2}\right)$ propuestos por Romero Zarco (1986). El índice $A_{1}$ es una estimación de la asimetría intracromosómica debida a la relación entre los brazos de cada par de cromosomas homólogos; y el $\mathrm{A}_{2}$ muestra la asimetría debida a la variación de tamaño en los cromosomas del cariotipo.

Se han realizado idiogramas utilizando los valores medios de los brazos de cada par de cromosomas homólogos de al menos cinco metafases. 


\begin{tabular}{|c|c|c|c|}
\hline $\mathrm{n}$ & $2 n$ & Origen del material & Autor(es) \\
\hline 6 & & Francia & SOUÉGES (1913) \\
\hline 8 & & España & LUQUE et al. (1988) \\
\hline 8,16 & & Suecia & LANGLET (1927) \\
\hline 12 & & Alemania & LOSCHNIGG (1925) \\
\hline 12 & & Alemania & WINKLER (1926) \\
\hline 16 & & Dinamarca & BÖCHER (1938) \\
\hline & 16 & Italia & CAPINERI et al. (1978) \\
\hline & 16 & Italia & FERRARELLA et al. (1979) \\
\hline & 16 & Dinamarca & LAEGAARD (1966) \\
\hline & 16 & España & LÖVE y KJELLQVIST (1974) \\
\hline & 16 & Italia & MICELI y GARBARI (1976) \\
\hline & 16 & España & VALDÉS-BERMEJO (1979) \\
\hline & 16 & Armenia, España, Italia, Polonia & POGAN y WCISLO (1986) \\
\hline & 16,32 & Austria & GREILHUBER (1974) \\
\hline & 16,32 & Reino Unido & HEYWOOD y WALKER (1961) \\
\hline & 16,32 & Reino Unido & MARCHANT y BRIGHTON (1971) \\
\hline & 16,32 & Polonia & POGAN y WCISLO (1973) \\
\hline & 16,32 & Francia y Yugoslavia & POGAN y WCISLO (1975) \\
\hline & $16,24,32$ & Portugal & BARROS NEVES (1942) \\
\hline & $16,24,32$ & Reino Unido & MARCHANT y BRIGHTON (1974) \\
\hline & $16,24,32$ & Reino Unido & NICHOLSON (1983) \\
\hline & $16,24,32$ & Bulgaria, Hungría, Polonia, Rumania & POGAN y WCISLO (1974) \\
\hline & $16,32,40$ & Italia & MARCHI et al. (1975) \\
\hline & $16+(0,1) B, 32$ & Reino Unido & JONES (1969) \\
\hline & $16+(0,2) B, 32$ & Desconocido & LARTER (1932) \\
\hline & $16+(0-8) \mathrm{B}, 32$ & Hungría & POGAN y WCISLO (1981) \\
\hline & $16+(0-7) \mathrm{B}, 24,32$ & Reino Unido & GILL et al. (1972) \\
\hline & 24,32 & U.R.S.S. & LAURENKO y SERDITOV (1986) \\
\hline & 24,32 & España, Portugal & POGAN y WCISLO (1986) \\
\hline & 32 & Holanda & ANDREAS (1954) \\
\hline & 32 & Alemania & $\operatorname{COOK}(1963)$ \\
\hline & 32 & Holanda & DELAY (1947) \\
\hline & 32 & Holanda & GADELLA y KLIPHUIS (1967) \\
\hline & 32 & Desconocido & GOEPFERT (1974) \\
\hline & 32 & Francia & HOCQUETTE (1922) \\
\hline & c. 32 & Italia & LARSEN y LAEGAARD (1971) \\
\hline & 32 & Yugoslavia & PAPES y TRINAJSTIC (1981) \\
\hline & 32 & Hungría & POLYA $(1950)$ \\
\hline & 32 & U.R.S.S. & SEMERENKO (1985) \\
\hline & 32 & Polonia & SKALINSKA et al. (1959) \\
\hline & 32 & Finlandia & SORSA (1963) \\
\hline & 32 & Reino Unido & WILCOX (en MARSDEN-JONES 1935 \\
\hline & 48 & Desconocido & RUTLAND (1941) \\
\hline
\end{tabular}

Tabla 1. Números cromosómicos para Ranunculus ficaria "sensu lato", según la bibliografía consultada. 
De cada población se han estudiado entre 5 y 10 ejemplares.

En todos los casos, los ejemplares utilizados se indican por medio del número de pliego con que se conservan en el Herbario del Departamento de Biología Vegetal y Ecología de la Facultad de Biología de Sevilla (SEV).

\section{RESULTADOS}

ALAVA: Ordoñana, robledal, 25-V-1988, Díaz, Diosdado y Pérez (SEV 128146), 2n=16. CADIZ: Jerez de la Frontera, Estella del Marqués, 6-II-1987, Diosdado (SEV 128143), $\mathrm{n}=8$, $2 \mathrm{n}=16$. Grazalema, Llanos de la'Camilla, 18II-1988, García Esteban (SEV 128138), 2n=16. Los Barrios, Montera del Torero, 18-IV-1989, Diosdado (SEV 128144), 2n=16. HUELVA: Navahermosa, 23-V-1989, Diosdado (SEV 128139), 2n=16. JAÉN: Sierra de Cazorla, base del Cabañas, 1800 m.s.m., 11-V-1989, Diosdado y Vioque (SEV 128140), 2n=16. MALAGA: La Sauceda, 20-III-1988, Diosdado (SEV 128145), $n=8,2 n=16$. SEVILLA. Coripe, Peñón de Zaframagón, 23-III-1988, Diosdado y Pérez (SEV 128141), 2n=16. CACERES: Baños de Montemayor, 28-IV-1988, Diosdado y Vioque (SEV 128147), 2n=24. ALMERIA: entre Laujar y Alcolea, 10-V-1989, Diosdado y Vioque (SEV 128142), 2n=32.

Los números encontrados en el presente estudio, tanto el gamético $\mathrm{n}=8$, como los somáticos $2 n=16,24$ y 32 , coinciden con la mayoría de los numerosos datos bibliográficos existentes (tab. 1).

En los diploides los cromosomas son entre medianamente grandes y grandes, oscilando el tamaño aparente de los cromosomas de 6'07 a 11'07 $\mu \mathrm{m}$ en la muestra de La Sauceda, de 6'25 a 12' $14 \mu \mathrm{m}$ en la de Coripe, de 6' 10 a 12 ' $59 \mu \mathrm{m}$ en la de la Montera del Torero y de 6' 15 a 11'70 $\mu \mathrm{m}$ en la de Ordoñana. El grado de asimetría es $2 \mathrm{~A}$ y los índices de asimetría presentan los valores: $A_{1}=0$ '51 y $A_{2}=0$ '22. Sus cariogramas apenas muestran diferencias. En la muestra de Coripe (SEV 128141) la fórmula del cariograma (Fig. 1, A) es: $2 \mathrm{M}+2 \mathrm{~m}+2(\mathrm{~m}-$ $\mathrm{sm})+2 \mathrm{sm}+2(\mathrm{sm}-\mathrm{st})+4 \mathrm{st}+2 \mathrm{t}^{\mathrm{sat}}$. En la población procedente de La Sauceda (SEV 128145) el cariograma (Fig. 1, B) presenta la fórmula: $2(\mathrm{M}-\mathrm{m})+4 \mathrm{~m}+2 \mathrm{sm}+2(\mathrm{sm}-\mathrm{st})+4 \mathrm{st}+2 \mathrm{t}^{\mathrm{sat}}$. Los valores medios de los brazos de cada par de cromosomas obtenidos en las poblaciones diploides, permiten elaborar un idiograma (Fig. 4, A) cuya fórmula está compuesta por: 2(M$m)+2 m+2(m-s m)+2 s m+6 s t+2 t^{\text {sat }}$. Las meiosis observadas en los diploides presentan siempre 8 bivalentes.

En el triploide, el tamaño aparente de los cromosomas varía entre 5'07 y 12'14 $\mu \mathrm{m}$, considerándose entre medianamente grandes y grandes. El grado de asimetría es $3 \mathrm{~B}$ y los índices de asimetría son: $\mathrm{A}_{1}=0$ ' 48 y $\mathrm{A}_{2}=0$ '24. $\mathrm{Su}$ cariograma (Fig. 2, B) presenta los cromosomas en grupos de tres y dispuestos según la fórmula: $3(\mathrm{M}-\mathrm{m})+6 \mathrm{~m}+3 \mathrm{sm}+3(\mathrm{sm}-$ $\mathrm{st})+6 \mathrm{st}+3 \mathrm{t}^{\mathrm{sat}}$. Del estudio de varias placas metafásicas resultan los valores medios de los brazos de cada grupo de cromosomas. De ellos se obtiene para los ejemplares triploides el idiograma (Fig. 4, B) cuya fórmula es idéntica a la del cariograma: $3(\mathrm{M}-\mathrm{m})+6 \mathrm{~m}+3 \mathrm{sm}+3(\mathrm{sm}-$ $\mathrm{st})+6 \mathrm{st}+3 \mathrm{st}^{\mathrm{sat}}$.

En el tetraploide, los cromosomas son entre medianamente pequeños y grandes pues el tamaño aparente oscila entre 4'77 y 12'78 $\mu \mathrm{m}$. El grado de asimetría es 3B y los índices de asimetría presentan los siguientes valores: $\mathrm{A}_{1}=0$ '53 y $\mathrm{A}_{2}=0$ '27. La fórmula del cariograma (Fig. 3, B) es en este caso: $12 \mathrm{~m}+4 \mathrm{sm}+4$ (sm$\mathrm{st})+8 \mathrm{st}+4 \mathrm{t}^{\text {sat }}$. Los valores medios, en el caso del tetraploide, se han realizado por separado para las dos parejas de cromosomas que forman un grupo, pues algunos presentan diferencias de tamaño (grupos 1, 2 y 6) o morfología (grupo 7) entre ambos pares. Se trata de una clara tendencia hacia un proceso de diploidización, con el consiguiente incremento de la formación de bivalentes en meiosis, lo 
1

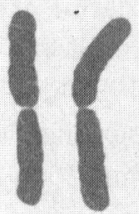

M

5

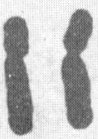

sm
2

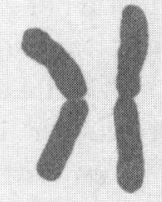

m

6

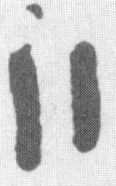

$t^{\text {sat }}$
3

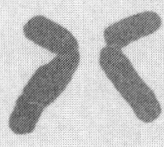

m-sm

7

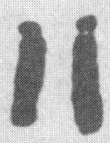

st
4

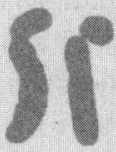

sm-st

8

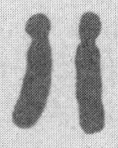

st

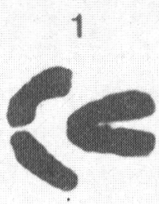

$M-m$
2

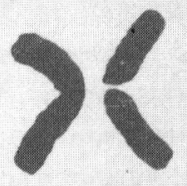

m

6

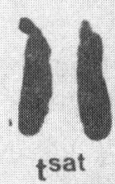

3

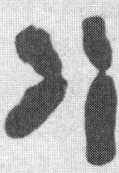

m
4

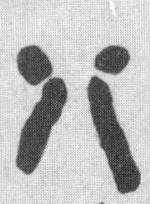

sm-st

5

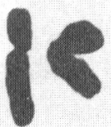

sm

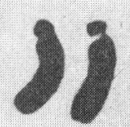

st
8

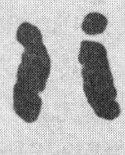

st 
cual produce un aumento de la fertilidad. Sin embargo el hecho de que la mayoría de los grupos presenten cromosomas homólogos, junto con una reproducción preferentemente apomíctica mediante bulbillos axilares, permite conferirle un carácter relativamente reciente a dicha tendencia a la diploidización. El idiograma obtenido en los ejemplares tetraploides (Fig. 4, C) presenta la fórmula: $12 m+6 s m+2(s m-s t)+8 s t+4 t^{\text {sat }}$.

\section{DISCUSIÓN}

Anteriormente varios autores han indicado cariogramas para $R$. ficaria. Larter (1932) señaló que el complemento diploide de esta especie consiste en 4 pares con el centrómero en la región media, 1 par con el centrómero submediano y 3 pares con constricción subterminal, presentando uno de ellos satélites y pudiendo considerarse telocéntrico. Barros Neves (1942) indicó una fórmula similar a la Larter (1. c.) señalando que en los triploides y tetraploides los cromosomas se reparten en los mismos tipos cromosómicos que en los diploides. Goepfert (1974) observó para los tetraploides unas posiciones relativas del centrómero a las que, de acuerdo con la terminología de Levan et al. (1964), correspondería la siguiente fórmula idiogramática: $12 m+4 s m+12 s t+4 t$. Pogan \& Wcislo (1975) indicaron para diploides pertenecientes a las subespecies ficaria y calthifolius el mismo complemento cromosómico formado por 3 pares con el centrómero en la región media (m), 2 pares con el centrómero en la región submedia (sm), 1 par con el centrómero en la región subterminal (st) y 2 pares con el centrómero en la región terminal (t), estando uno de ellos satelizado. Para los tetraploides, pertenecientes a la subsp. bulbifer, citó los mismos tipos cromosómicos que en los diploides. Marchi et al. (1975) muestran los cariogramas de plantas diploides, tetraploides y pentaploides de donde se deduce, por la terminología de Levan et al. (1. c.), la siguiente fórmula gamética: $3 m+1 s m+3 s t+1 t^{\text {sat }}$, que al multiplicarla por 2, 4 ó 5 define el cariograma diploide, tetraploide y pentaploide respectivamente. Ferrarella et al. (1979) indicaron para la subsp. ficaria un idiograma de donde se deduce la fórmula $4 m+4 s m+2(s m-s t)+4 s t+2 t$, sin hacer referencia a la presencia de cromosomas satelizados.

Los datos aportados en el presente estudio son muy similares a los mencionados por Goepfert (1974), Marchi et al.(1975) y Ferrarella et al. (1979), que pueden englobarse en la fórmula gamética: (3-2) $\mathrm{m}+(1$ 2) $s m+3 s t+1 t$. Sin embargo, Larter (1932) y Barros Neves (1942) indican 4 pares de cromosomas metacéntricos, mientras que Pogan \& Wcislo (1.c.) señalan la existencia de 2 pares de cromosomas telocéntricos. En el presente estudio, no se han observado los cromosomas supernumerarios citados en algunas poblaciones de plantas diploides (Jones, 1969; Gill et al. 1972). Por los datos obtenidos y los reseñados por los autores consultados, $R$. ficaria presenta un número básico $\mathrm{x}=8$.

De acuerdo con las placas metafásicas observadas, $R$. ficaria muestra un predominio de cromosomas metacéntricos (m) con el centrómero en la región media y subtelocéntricos con el centrómero en la región subterminal (st), con un 31'73\% y 30 ' $30 \%$ respectivamente. Siguen los submetacéntricos con el centrómero en la región submedia (sm) que suponen un 19'70\%. En porcentajes menores se encuentran los metacéntricos con el centrómero en el punto medio (M) y los telocéntricos con el centrómero en la región terminal (t), con un 5'77\% y $12^{\prime} 50 \%$ respectivamente.

Esta especie presenta, en su área de distribución, una serie poliploide compuesta desde el nivel diploide hasta el hexaploide. En la Península Ibérica sólo se han encontrado los niveles $2 \mathrm{x}, 3 \mathrm{x}$ y $4 \mathrm{x}$, con predominio del prime- 


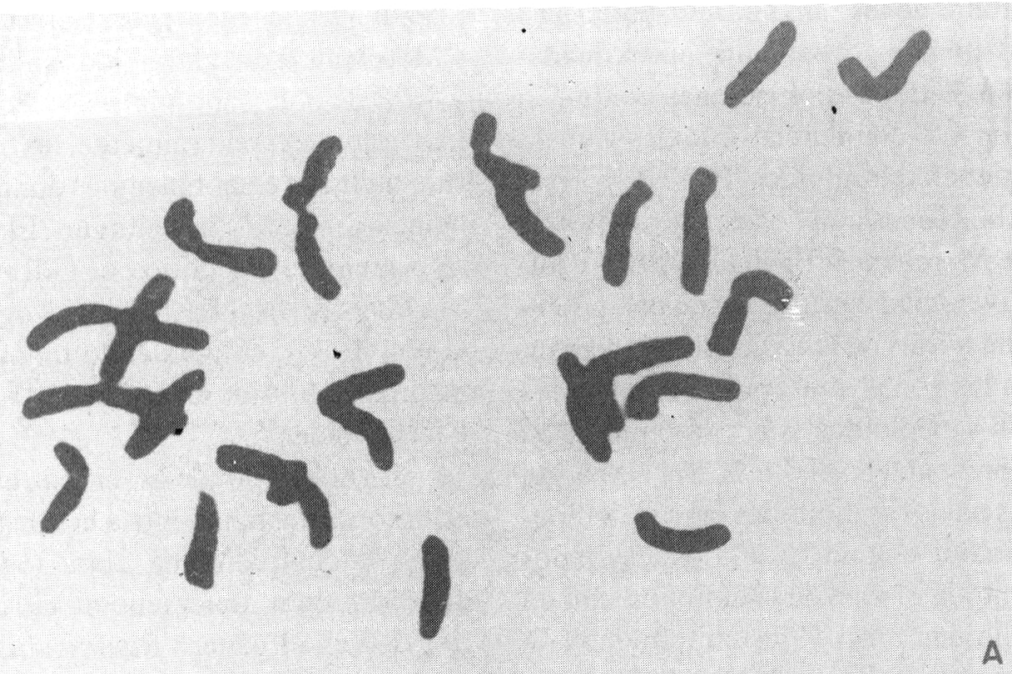

1

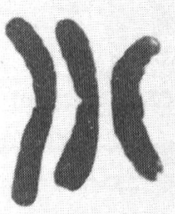

M-m

5

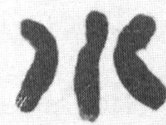

sm
2

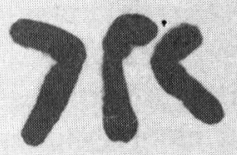

m

6

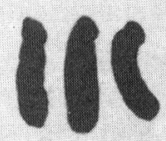

st

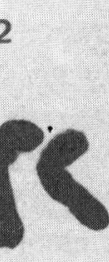

3

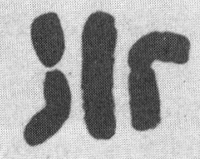

m

7

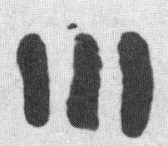

$t^{\text {sat }}$

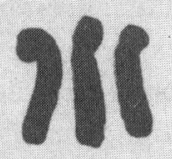

sm-st

8

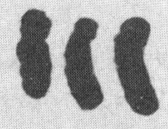

st

B 
ro sobre los otros dos. Gill et al. (1972), en un extenso estudio de las razas cromosómicas en las Islas Británicas, citan que aproximadamente un $66 \%$ de las poblaciones contienen diploides, un $4 \%$ contienen triploides y un 43 $\%$ que contienen tetraploides. El $13 \%$ corresponde a poblaciones con dos o más niveles de poliploidía. Marchant \& Brighton (1974) estudiaron la diversidad cariológica en una población cercana a Survey (Inglaterra) y observaron que, en las zonas altas, predominaban los tetraploides y triploides sobre los diploides, que sólo representaban el 6' $4 \%$. Sin embargo, en las zonas bajas los diploides eran el componente dominante con un $72 \%$. Los resultados globales en toda el área de estudio indican un $31 \%$ de diploides, un $40 \%$ de tetraploides y un $29 \%$ de triploides. Nicholson (1983) en su estudio sobre la distribución y relación entre las razas cromosómicas de $R$. ficaria en el sureste de Yorkshire (Inglaterra) encontró que de 96 poblaciones investigadas, 57 contenían sólo plantas diploides, 26 sólo tetraploides y las 13 poblaciones restantes presentaban plantas diploides, triploides y tetraploides. Por tanto, los porcentajes de los distintos niveles de ploidía, aún dependiendo de la zona estudiada, muestran un aumento de los poliploides en áreas donde la subsp. bulbilifer (tetraploide) es más abundante, es decir, en el norte, centro y este de Europa. En la Península Ibérica, la subespecie bulbilifer no resulta muy abundante, aunque su distribución es conocida de forma imperfecta (López González, 1986). Esto explicaría el bajo porcentaje registrado de poliploides.

El origen del triploide es controvertido. En contraposición con los triploides provistos de bulbillos axilares encontrados en Gran Bretaña (Gill et al., 1972 y Marchant \& Brighton, 1974) y Polonia (Pogan \& Wcislo, 1974) los observados por Barros Neves (1942) en Portugal y los del presente estudio en una población de Baños de Montemayor (Cáceres) no presentan esos bulbillos, a pesar de no producir aquenios fértiles, debido seguramente a sus irregularidades meióticas. Barros Neves (1. c.) y Marchant \& Brighton (1. c.) indican un $77 \%$ y $80 \%$ de polen anormal, respectivamente, en los triploides. Generalmente, los triploides han sido hallados en poblaciones donde coexistían plantas diploide y tetraploides. El problema de su origen fue discutido por Gill et al. (1972), Marchant \& Brighton (1974) y Pogan \& Wcislo (1974, 1975), coincidiendo todos ellos en su naturaleza híbrida a partir de táxones diploide y tetraploides.

En el tetraploide observado en el presente estudio, correspondiente a la subsp. bulbilifer, se ha encontrado una clara tendencia a la diploidización, fenómeno no citado con anterioridad en el género Ranunculus, aunque es conocido en otros géneros, así Fernandes (1969) indica la formación de bivalentes en varios poliploides de Narcissus, Wet \& Harlan (1970) encontraron que en la serie poliploide (2x, 4x y 6x) de Dichanthium sólo se formaban bivalentes, y Pastor $(1982,1985)$ señaló procesos de diploidización en Allium.

Las plantas diploides presentan un grado de asimetría del tipo $2 \mathrm{~A}$, mientras que en los triploides y tetraploides es 3B. De acuerdo con Pogan \& Wcislo (1975) los táxones diploides son los más primitivos, presentando aquenios fértiles en un porcentaje elevado, mientras que los tetraploides son más evolucionados, presentando una reproducción exclusivamente vegetativa por bulbillos axilares y raíces engrosadas.

Al presentar los cariogramas de los diploides, triploides y tetraploides los mismos tipos cromosómicos, parece ser que la poliploidización es uno de los principales mecanismos de evolución de R. ficaria. Aunque también ha intervenido la hibridación entre diploides y tetraploides, además de fenómenos de mutación y recombinación que, sin duda, contribuyen a un incremento de la variabilidad de la especie «sensu lato» (Pogan \& Wcislo, 1.c.). 


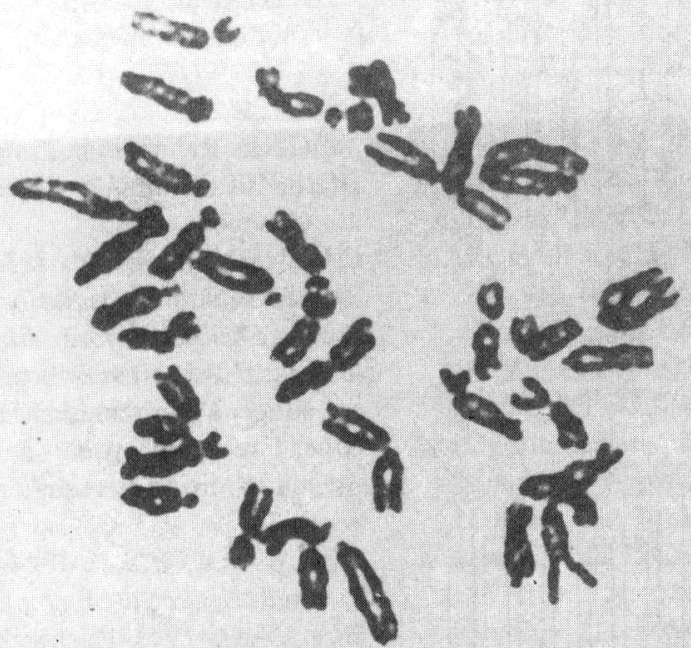

1

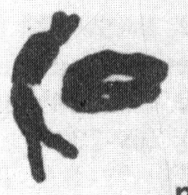

m

3

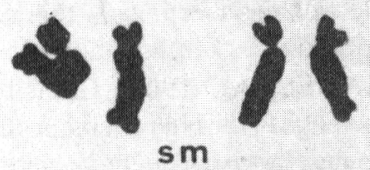

5

$7:$

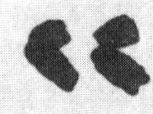

m

7

81

sm-st
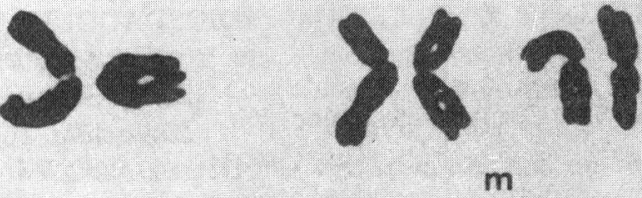

4

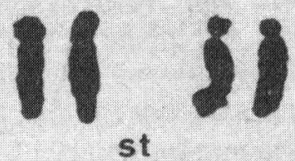

6
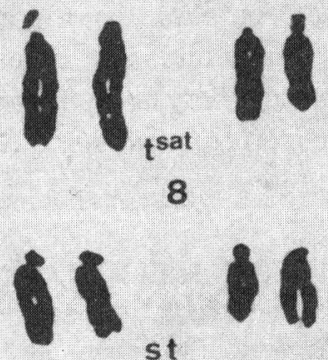

B

Figura 3. Metafase somática y cariograma de $R$. ficaria (4x) (Almería, 128142). 

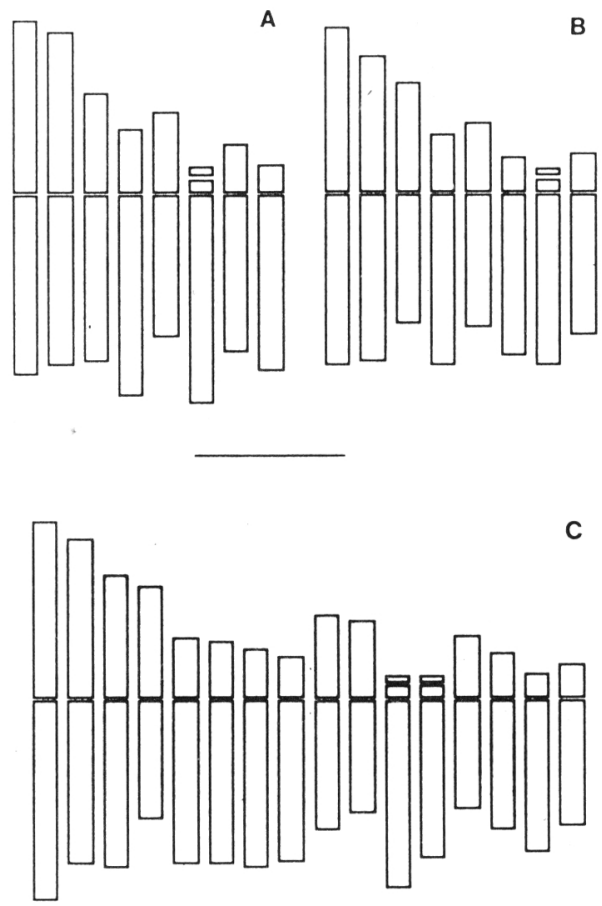

Figura 4. Idiogramas de $R$. ficaria. A, nivel diploide $(2 \mathrm{x})$; $\mathrm{B}$, nivel triploide (3x); C, nivel tetraploide $(4 x)$.

Comparando los idiogramas obtenidos en $R$. ficaria con otras especies del género se encuentra relación con algunos táxones de las secciones Ranunculastrum y Chrysanthe, como ya indicaron anteriormente Larter (1932), Barros Neves (1942), Goepfert (1974) y Diosdado y Pastor (1990, 1992). Así pues, los estudios cariológicos reforzarían la inclusión de este taxón en el género Ranunculus y no justificaría su separación en el género Ficaria como han propuesto algunos autores.

\section{BIBLIOGRAFÍA}

ANDREAS, C.H. -1954- Notes on Ranunculus ficaria L. in the Netherlands. Acta Bot. Netherl., 3: 446-453.

BARROS NEVES, J. de -1942- Sobre a cariologia de R. ficaria L. Bol. Soc. Brot. Ser., 2, 16: 169181.

BENSON, L. -1940- The North American subdivisons of Ranunculus. Am. Journ. Bot., 27: 799-807.

BÖCHER, T.W. -1938- Cytological studies in the Genus Ranunculus. Dansk Bot. Ark., 9: 1-33.

BOISSIER, E. -1867- Flora Orientalis, 1. Basel, Genève.

CAPINERI, R., G. D'AMATO \& P. MARCHI 1978- Numeri cromosomici per la flora italiana: 543-583. Inform. Bot. Ital., 10: 421-465.

COOK, C.D.K. -1963- Studies on Ranunculus L. subgenus Batrachium (DC.) A. Gray, II. General morphological considerations in the taxonomy of the subgenus. Watsonia, 5: 294303.

DECANDOLLE, A.P.-1824-Prodromus systematis naturalis regni vegetabilis, 1 . Paris.

DELAY, C. -1947- Recherches sur la structure des noyaux quiescents chez les phanérogames. Rev. Cytol. et Cytophys. Végét., 9: 189-223; 10: 103-229.

DIOSDADO, J.C. y J. PASTOR -1990- Estudio cariosistemático del género Ranunculus L. sect. Ranunculastrum DC. en la Península Ibérica. Lagascalia, 16 (2): 269-290.

DIOSDADO, J.C. y J. PASTOR -1992Citotaxonomía de las especies vivaces del género Ranunculus L. sect. Chrysanthe (Spach) L. Benson en la Península Ibérica. Candollea, 47: 555-576.

FERNANDES, A. -1969- Contribution to the knowledge of the biosystematic of some species of genus Narcissus L. In: V. Simposio de Flora Europaea p.245-284.- Sevilla.

FERRARELLA, A., I. FIORE, M.G. DIA \& N. ALLIATA -1979- Numeri cromosomici per la flora italiana: 647-651. Inform. Bot. Ital. 11: 301-305.

FREYN, J. F. -1880- Ficaria L., in H. M. WILLKOMM \& J. LANGE (eds.) Prodromus Florae Hispanicae, 3: 942-943. Stuttgart.

GADELLA, T.W.J. \& K. KLIPHUIS -1967Chromosome numbers of flowering plants in the Netherlands III. Koninkl. Nederl. Akademie Wet. Amsterdan, Proc. Ser. C, 70: 7-20.

GILL, J.J., B.M. JONES, C.J. MARCHANT, M.J. MCLEISH \& D.J. OCKENDON -1972- The distribution of chromosome races of Ranunculus 
ficaria L. in the British Isles. Ann. Bot., London, 36: 31-47.

GOEPFERT, D. -1974- Karyotypes and DNA content in species of Ranunculus $\mathrm{L}$. and related genera. Bot. Not., 127: 464-489.

GREILHUBER, J. -1974- Ein chromosomensatz von R. ficaria subsp. calthifolius. Mitt. Bot. Arbeitsgem. Oberösterr. Landesmus. Linz 6: 3-6.

HALLER, A. von -1742- Enumeratio methodica stirpium Helvetiae indigenarum, 1. Göttingae.

HEYWOOD, V.H. \& S. WALKER -1961Morphological separation of cytological races in Ranunculus ficaria L. Nature 189: 604.

HOCQUETTE, W. - 1922- Observations sur le nombre des chromosomes chez quelques Ranunculacées. Compt. Rend.Soc. Biol. France, 87: 1301-1303.

JONES, B. G. -1969- The morphology of polyplotypes of Ranunculus ficaria L. Biol. Jour. Linn. Soc., 1: 10-11.

JUSSIEU, A. L. -1789- Genera Plantarum. Paris. LAEGAARD, S. -1966- R. ficaria subsp. fertilis in Denmark. Bot Tidskr., 21: 1-17.

LANGLET, O.F.J. -1927- Beitrage zur zytologie der Ranunculaceen. Svensk. Bot. Tidskr., 21: 117.

LARSEN, K. \& S. LAEGAARD -1971Chromosome studies of the sicilian flora. Bot. Tidsskr., 66: 249-268.

LARTER, L.N. -1932-Chromosome variation and behaviour in Ranunculus L. Journ. Genet., 26: 255-283.

LAURENKO, A.N. \& N.P. SERDITOV -1986Chromosome numbers in some representatives of Ranunculaceae, Paeoniaceae, Boraginaceae families from the North of the European of the USSR and the Urals. Bot. Zur., 71: 1694-1695.

LEVAN, A., K. FREDGA \& A.A. SANDBERG 1964- Nomenclature for centromeric position on chromosomes. Hereditas, 52: 201-220.

LOPEZ GONZALEZ, G. -1986- Ranunculus L. en S. CASTROVIEJO et al. (eds.). Flora Ibérica, 1: 298-301; 310-353.

LOSCHNNIG, F. -1925- Bausteine zu einer Monographie von Ficaria. 4. Über die Ursachen der häufigen sterilität. Beiträge Biol. Pflanzen, 14: 347-358.

LÖVE, A. \& E. KJELLQVIST -1974Cytotaxonomy of spanish plants. III \& IV:
Salicaceae - Rosaceae, Caesalpinaceae Asteraceae. Lagascalia, 4: 3-32 y 152-211.

LÖVE, A. \& D. LÖVE-1975-Plant chromosomes. Vaduz.

LUQUE, T., J.A. MEJIAS y Z. DIAZ LIFANTE 1988- Números cromosómicos para la flora española: 544-550. Lagascalia, 15: 130-133.

MAIRE, R. -1964- Flore de l'A frique du Nord. 11: 112-197. Paris

MARCHANT, C.J. \& C.A. BRIGHTON -1971Mitotic instability in the short Arm of a heteromorphic SAT Chromosome of tetraploid Ranunculus ficaria L. Cromosoma, 34: 1-18.

MARCHANT, C.J. \& C.A. BRIGHTON -1974Cytological diversity and triploid frequency in a complex population of $R$. ficaria L. Ann. Bot. London, 38: 7-15.

MARCHI, P., R. CAPINERI \& G. D'AMATO 1975- Numeri cromosomici per la flora italiana: 208-218. Inform. Bot. Ital., 7: 377-389.

MARDSEN-JONES, E.M. -1935- Ranunculus ficaria L.: life history and pollination. Journ. Linn. Soc. London, 50: 39-57.

MICELI, P. \& F. GARBARI -1976- Numeri cromosomici per la flora italiana: 255-262. Inform. Bot. Ital., 8: 207-216.

NICHOLSON, G.G. -1983- Studies on the distribution and the relationship between the chromosome races of Ranunculus ficaria $\mathrm{L}$. in S.E. Yorkshire. Watsonia, 14: 321-328.

OVCZINNIKOV, P.N. -1937- Ranunculus L. y Ficaria Dill., in V. L. KOMAROV (ed.) Flora of the USSR. VII. Leningrad.

PAPES, D. \& I. TRINASJSTIC -1981- In A. LÖVE (ed.) IOPB Chromosome number reports, LXX. Taxon, 30: 70.

PASTOR, J. -1982- Karyology of Allium species from the Iberian Peninsula. Phyton (Austria), 22 (2): 171-200.

PASTOR, J. -1985- Karyology of Allium stearnii and $A$. reconditum, two new species from the Iberian Peninsula. Phyton (Austria), 25 (1): 7376.

POGAN, E. \& H. WCISLO -1973- Studies in R. ficaria L., I. Karyological analysis of R. ficaria L. subsp. bulbifer (Marsden-Jones) Lawalrée and R. ficaria L. subsp. calthifolius (Rchb.) Arc. Acta Biol. Cracov., Sér. Bot., 16:135-143.

POGAN, E. \& H. WCISLO -1974- Studies in R. ficaria L., II. Further karyological studies. Acta 
Biol. Cracov., Sér. Bot., 17: 165-173.

POGAN, E. \& H. WCISLO -1975- Studies in $R$. ficaria L., III. Karyotype analysis. Acta Biol. Cracov., Sér. Bot., 18: 79-99.

POGAN, E. \& H. WCISLO -1981- Studies in $R$. ficaria L., V. Cytoembryological analysis of R. ficaria L. subsp. calthifolius (Rchb.) Arc. with B-chromosomes. Acta Biol. Cracov., Sér. Bot., 23: 83-96.

POGAN, E. \& H. WCISLO -1986- Studies in $R$. ficaria L., VII. Additions to chromosome numbers. Acta Biol. Cracov., Sér. Bot., 28: 87 92.

POLYA, L. -1950- Magyarorszagi noevenyfajok kromoszomaszamai. II. Ann. Biol. Univ. Debrecen, 1: 46-56.

ROMERO ZARCO, C. -1986- A new method for estimating karyotype asymmetry. Taxon, 35: 526-530.

RUTLAND, J. P. -1941- The Merton catalogue. A list of chromosome numbres of British plants. New Phythol., 40: 210.

SCHAEFFER, J.C. -1760- Botanica expeditior, 1. Regensburg.

SEMERENKO, L.V. -1985-Chromosome numbers in some species of flowering plants of Byelorusian Flora. Bot. Zur., 70: 992-994.

SKALINSKA, M., R. CZAPIK, M. PIOTROWICZ et al. -1959- Furthe studies in chromosome numbers of Polish Angiosperms (Dicotyledons). Acta Soc. Bot. Poloniae, 28: 487-529.

SNOW, R. -1963- Alcoholic hydrochloric acidcarmine as a stain for chromosomes in squash preparations. Stain Technol., 38: 9-13.

SORSA, V. -1963-Chromosomenzahlen Finnischer kormophyten. II. Ann. Acad. Sci. Fennicae, 4 Biol. 68: 1-14.

SOUÉGES, M.R. -1913- Recherches sur l'embryogénie de Ranunculaceén. Bull. Soc. Bot. France, 60: 150-157.

SPACH, E. -1839- Histoire naturelle des vegetaux. Phanerogames, 7: 191-220. Paris.

STEBBINS, G.L. -1938- Cytological characteristics associated with the different growth habits in the dicotyledons. Am. Journ. Bot., 25: 189-198.

STEBBINS, G.L. -1971- Chromosomal evolution in higher plants. London.

TAMURA, M. -1967- Morphology, ecology and phylogeny of the Ranunculaceae. VII. Sci. Rep. Osaka Univ., 16: 21-43.
TJIO, J.J. \& A. LEVAN -1950. The use of oxyquinoleine in chromosome analysis. Anal. Est. Exper. Aula Dei, 2: 21-64.

TUTIN, T.G. (1964)- Ranunculus L., in T. G. TUTIN et al. (eds.) Flora Europaea, 1: 223237. Cambridge.

VALDÉS, B. -1987-Ranunculus L., en B. VALDÉS et al. (eds.) Flora Vascular de Andalucía Occidental, 1: 97-114. Barcelona.

VALDÉS-BERMEJO, E. -1979- Números cromosomáticos de plantas occidentales: 1-34. Anal. Jard. Bot. Madrid, 36: 373-389.

WET, J.M. \& J.R. HARLAN -1970- Apomixis, polyploidy and speciation in Dichanthium. Evolution, 24: 270-277.

WINKLER, H. -1926- Bausteine zu einer Monographie von Ficaria. Beitr. Biol. Pflanzen, 14: 3383-3455.

Aceptado para su publicación en Abril de 1993

Dirección de los autores. Departamento de Biología Vegetal y Ecología, Universidad de Sevilla, Aptdo. 1095, 41080 Sevilla. 